SLAC-PUB-8167

July 1999

\title{
The IFR Online Detector Control system at the BaBar experiment
}

By P. Paolucci et al.

Presented at the Santa Fe 99, IEEE NPSS Real Time conference, 6/14/99ã6/18/99, Santa Fe, NM, USA 


\title{
The IFR Online Detector Control at the BaBar experiment
}

\author{
P.Paolucci $^{1}$, N.Cavallo ${ }^{2}$, F.Fabozzi ${ }^{2}$ and D.Piccolo ${ }^{2}$ \\ ${ }^{1}$ Stanford Linear Acceleretor Center, P.O. Box 4349, Stanford CA, 94309. \\ 2 I.N.F.N. Sezione di Napoli, Complesso Monte Sant'Angelo, 80126 Napoli, Italy.
}

\begin{abstract}
The Instrumented Flux Return (IFR)[1] is one of the five subdetectors of the BaBar[2] experiment on the PEP II accelerator at SLAC. The IFR consists of 774 Resistive Plate Chamber (RPC) detectors, covering an area of about 2,000 $\mathrm{m}^{2}$ and equipped with 3,000 Front-end Electronic Cards (FEC) reading about 50,000 channels (readout strips).

The first aim of a B-factory experiment is to run continuously without any interruption and then the Detector Control system plays a very important role in order to reduce the dead-time due to the hardware problems.

The I.N.F.N. group of Naples has designed and built the IFR Online Detector Control system (IODC)[3] in order to control and monitor the operation of this large number of detectors and of all the IFR subsystems: High Voltage, Low Voltage, Gas system, Trigger and DAQ crates.

The IODC consists of 8 custom DAQ stations, placed around the detector and one central DAQ station based on VME technology and placed in electronic house.

The IODC use VxWorks and EPICS to implement slow control data flow of about 2500 hardware channels and to develop part of the readout module consisting in about 3500 records. EPICS is interfaced with the BaBar Run Control through the Component Proxy and with the BaBar database (Objectivity) through the Archiver and KeyLookup processes.
\end{abstract}

\section{INTRODUCTION}

The IFR Online Detector Control system (figure 1) is required to:

- Monitor the operation of the RPC detectors and of their Front-end Electronic Cards (FEC) [4]:

- Measuring the Single Counting Rate and the Dark Current of each RPC;

- Monitoring the LV system of the FECs;

- Control and monitor the HV system;

- Monitor the RPC Gas system;

- Monitor the IFR trigger system;

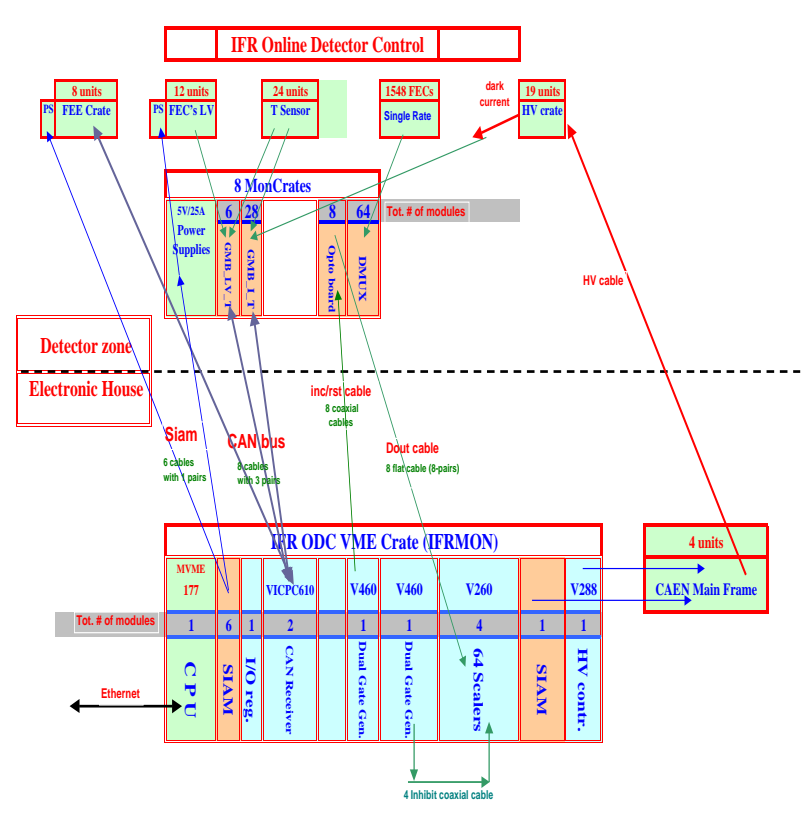

Figure: 1 General scheme of the IFR ODC system. The detector zone with the hardware input channels and the local DAQ system (MonCrate) are shown in the upper part. The central VME DAQ system is shown in the lower part.

- Control and monitor the IFR DAQ crates;

The core of the DAQ system is a $6 \mathrm{U}$ VME crate equipped with a Motorola CPU MVME177. It is located in the electronic house and receives data from the 8 local IODC subsystems, called MonCrate and placed on the detector (see figure 2).

All the analog signals (dark current, LV, FEE and Gas system) are before digitized and then sent to the central system via CAN-bus. The digital signals (RPC and Trigger rates) are discriminated and converted into the MonCrate and then sent to the VME crate via a twisted cable using opto-coupled ECL differential signals.

\section{IFR ODC SUBSYSTEMS}

\section{A. Monitor Crate}

The MonCrate is an EUROCARD 3U crate (figure3) equipped with a custom backplane, designed and build by the 
General scheme of the IFR system

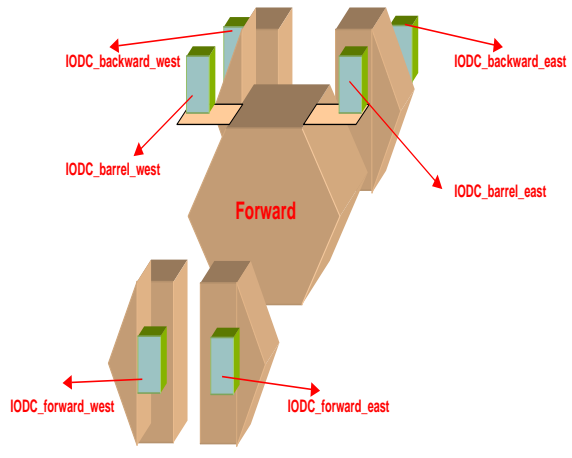

Figure: 2 The six independent DAQ subsystems located on the BaBar detector. Two of them are placed in the Barrel region and one on each endcap half door

I.N.F.N. group of Naples.

It is the remote, not accessible and opto-coupled slow control DAQ system, housing the digital and analog DAQ cards (see fig. 1).

The local system provides the following measurements:

- RPC Single counting Rate (digital signals);

- RPC Dark Current (analog signals);

- LV, Gas and Temperature (analog signals);

Each crate houses 2 power supplies ( $+5 \mathrm{~V} / 2 \mathrm{~A}$ and $-5.2 \mathrm{~V} / 2 \mathrm{~A})$, 8 multiplexer cards, 1 "opto-coupled" board and 8 General Monitor Board[5].

\section{B. Single Counting Rate}

Every time, at least one of the RPC strips is fired (noise, cosmics or physics hits), the FEC provides a fast-OR pulse output. The rate of the fast-OR is defined as the RPC single counting rate. The single counting rate is a very interesting quantity because it depends on the RPC HV operating value, on the gas mixture and on the RPC temperature. Monitoring continuously it allows us to check the "good" and "stable" performances of the IFR system.

The total number of fast-OR to acquire is 1548. These signals are at least $10 \mathrm{nsec}$ wide and have a rate of about 3 KHz. The DAQ system has to be able to count these signals for a time interval of 10 seconds in order to measure it with an error less than $1 \%$.

The I.N.F.N. group of Naples has designed and built a digital multiplexer (Dmux) in order to reduce the number of channels to acquire. The Dmux is a $3 \mathrm{U}$ board with 32 differential ECL input signals and one output channel. Two NIM input signals are used to increment and reset the address.

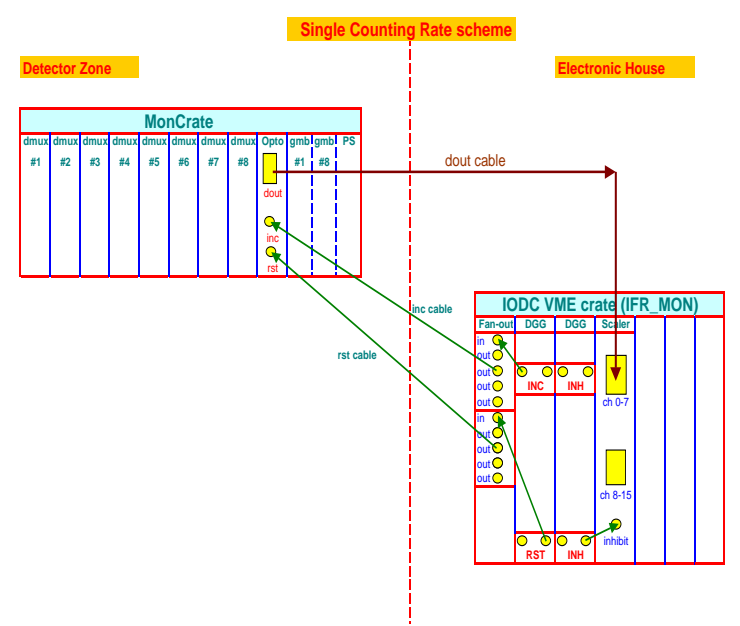

Figure: 3 The single counting rate scheme is shown. The MonCrate (left side) comunicates with the central VME crate (right side) placed in the electronic house through a set of opto-coupled ECL and NIM signals.

The multiplexer stage is based on a single TTL device: MACH 210 PLD. When the increment signal arrives, the PLD increments the actual channel number allowing the selected input to be sent out. Since the actual address is not read back, the reset signal is used to reset the PLD counter. The ECL input stage has a protection network, implemented with a bank of SP720 suppressor devices, in oder to prevent damages due to induced spark transients.

Each MonCrate houses 8 Dmuxs and 1 OptoBoard that provides the opto-coupling between the local DAQ crate and the electronic house. The eight Dmux output signals are sent to the OptoBoard, through the backplane, where are opto-coupled (HCPL 2601 high speed TTL compatible optocoupler) and sent to the VME scalers placed in the Electronic House.

A VME time unit (Dual Gate Generator), placed in the $\mathrm{EH}$, is used to generate the reset and increment signals. These two NIM signals are sent to the OptoBoard where are opto-coupled and sent, through the backplane, to the eight Dmuxs.

This system is able to acquire more than 1500 channels, coming from 8 different regions, in less than $100 \mathrm{sec}$. The general scheme of the single counting rate system is shown in figure 3 .

\section{HV system}

The IFR HV system consists of 4 CAEN mainframes SY127, equipped with the communication controller 128HS and housing up to $10 \mathrm{HV}$ plug-in modules mod. A330P. These modules provides two independent HV channels each with $+10 \mathrm{KV}$ and a maximum current of $1 \mathrm{~mA}$. The HV-current 
resolution is of about $3 \mathrm{~V} / 1 \mu \mathrm{A}$.

The four mainframes, placed in the electronic house, are connected in daisy-chain and are remotely controlled by a VME V288 HS CAENET controller. It is a send and receive system and permits a serial transmission of data packets along a 50 Ohm coaxial cable with a data transfer rate of 1 MBaud.

The standard parameters provided by the CAEN commands for each individual $\mathrm{HV}$ channel are the following:

- Two voltage values;

- Two current limit values;

- Maximum HV increase-decrease rate;

- Maximum time in overcurrent (trip);

- Voltage and current monitored values;

- Status (on, off, ovc, trip, ovv and unv).

\section{Analog Data}

The IFR system has about 900 analog channels to acquire, coming from the LV system, the Dark Current system and from the Temperature Transducer (AD592).

The BaBar collaboration has built a general purpose board, the General Monitor Board (GMB), and we decided to use it to acquire the analog information. The GMB is a custom board with 32 analog input channels, based on the Motorola Microcontroller MC68HC705X32 and interfaced to the BaBar standard serial bus, the CAN bus[6]. The IFR GMB has 30 input channels configured to read analog voltage (from 0 Volt to 4.096 Volts) and 2 channels configured to read the output signal of the AD592 transducer (current signal).

Each CAEN HV channel is splitted in 12 or 18 channels in the HV crates. These crates are placed around the detector and contain $6 \mathrm{HV}$ boxes. Each box provides $6 \mathrm{HV}$ output channels and 6 analog signals proportional to the dark current drawn by the single RPC detector.

The LV system consists of $8 \mathrm{LV}$ crates placed on the detector. Each crate is equipped with a monitor board adapting the monitor signals of the power supplies to the input requirements of the GMB boards. The voltage and the current of the 50 power supplies are monitored by $8 \mathrm{GMBs}$.

One GMB, placed in the gas house, is used to monitor IFR gas system. The variables monitored are: the pressure and the flow of the 3 gasses and the pressure and the flow of the gas mixture.

\section{E. CAN bus}

The IFR has 8 DAQ crates placed on the detector. They are the standard $\mathrm{BaBar}$ crate equipped with a custom backplane.

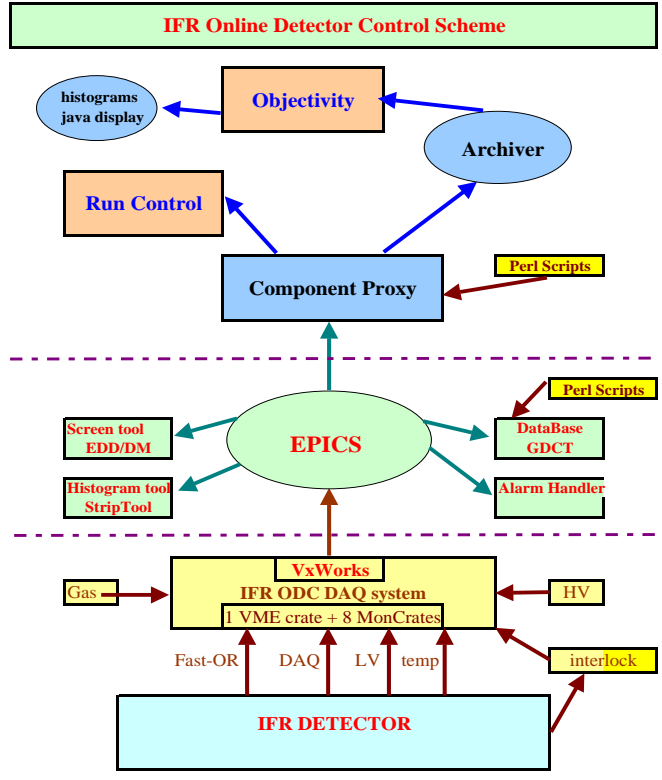

Figure: 4 The IODC software schema is shown here from the hardware system to the BaBar database, through the EPICS world

Each crate is equipped with a remote system, based on the CAN-bus protocol, allowing a complete monitor of the crate operations (LV, fan and status info).

The IFR ODC system has 6 CAN-bus lines housing up to 16 GMBs and 2 FEE crates plus one line used to read the GMB placed in the gas house.

The CAN bus data are controlled by two VIPC616 VMEbus IP carriers equipped with four TIP810 CAN-Bus interface using the Philips PCA82C200 CAN-Controller.

\section{Detector Control Software}

\section{A. EPICS system}

EPICS is a multi-layered distributed client-server control system package. It makes use of the VxWorks real time operating system for hardware access, TCP/IP for network communication, and workstations for development, operator display and interaction.

The IFR ODC system is divided in 3 independent components, called barrel, endcap and general from both the hardware and software point of view. The independence of the components allows to take data with just a part of the subdetector. The barrel and endcap components consist of 5 different subsystems (HV, LV, DAQ, Single Rate and Dark Current) while the general includes the Gas and the Temperature systems.

The IODC software package (see fig. 4) has been developed starting from the EPICS database. It consists of 2500 EPICS 


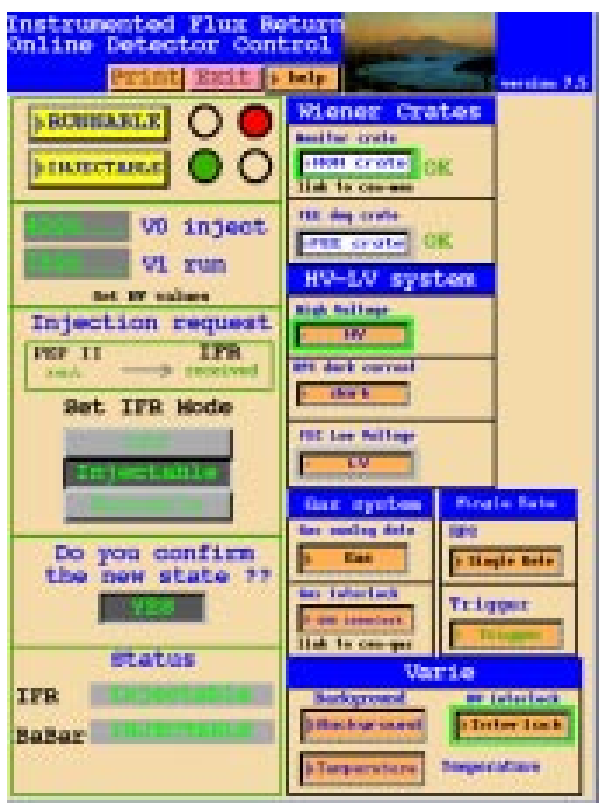

Figure: 5 The main IFR EPICS screen contains some general information coming from PEPII and BaBar, plus the status of each IFR subsystems. Each subsystems has a set of subscreens, reachable pushing its own button.

records corresponding to as many hardware channels plus 1000 records used to summarize the results and the status of each IFR subsystem.

Each record contains information as the scan period, the operating range the alarm limit, the alarm severity and the archive deadband. The scan period is the refresh rate of the hardware channel and the record value is updated only when the new value is over the deadband. When a value exceeds the alarm limit an alarm status is set following a hierarchy written in the alarm severity.

The sequential operations of each subsystem are managed by a real-time state machine, called sequencer and produced by the State Notation Language. They are part of the EPICS system and provide a very powerful tool for programming sequential operations in real-time when using different VME boards.

A graphical interface has been developed for each IODC subsystems using the EDD/DM EPICS tools. The main IFR screen (see figure. 5) contains some useful information coming from PEPII and the other BaBar subdetectors and the summary status of each IFR subsystem. The summary status of each subsystem is calculated by a sequencer making logical operations on the alarm status of the single records.

When the summary status of a subsystem become RED, the operator in shift can understand which is the record with an alarm going through the subscreens or looking at the BaBar
Alarm Handler that monitors all the alarm status.

\section{From EPICS TO THE BABAR RUN CONTROL AND DATABASE}

The interface between the EPCIS system, the BaBar Run Control and the Object-Oriented database are provided by the Component Proxy. They are unix tasks, implemented using cdev and the cdev Generic Server, running continuously.

When the run control sends a configuration transition the Component Proxy starts a coprocess, called KeyLookup, that read the "hardware setpoints" from the configuration database, translates them to cdevData objects and sends them to the Component proxy and so to the EPICS world.

Whenever an EPICS record is updated, the Component Proxy sends this new value to the Archiver coprocess, running continuously. The Archiver accumulates the new data in transient objects and every fixed period assigns them to persistent objects in the database.

The detector control data are read back from the database and are analyzed and displayed using the BaBar Java Browser.

\section{CONCLUSION}

The IODC has been installed during the 1998 and is working since the August 1998. All the data acquired during the October 1998 cosmic run and during the data tacking, started in May 1999, are written in the BaBar database and are used for the analysis data.

\section{REFERENCES}

[1] P.Paolucci et al., Nucl. Instr. and Meth. A 379 (1996) $472-$ 474.

[2] BaBar Technical Design Report, SLAC report \#95-457, 1995

[3] P. Paolucci, IFR Online Detector Control, BaBar Note \# 403 Jan 10, 1998

[4] N. Cavallo, P.Paolucci et al., Front-end readout development for the IFR muon detector at BaBar, Nucl. Instr. and Meth. A 409 (1998) 297-299

[5] W.T.Meyer and R.L.McKay, :The BaBar General Monitor Board, BaBar Note \# 366 May 7, 1998

[6] W.T.Meyer and R.L.McKay, : Using the Controller Area Network and the MC68HC705X32 Microcontroller in BaBar, BaBar Note \# 365 May 7, 1998 